\title{
Multiplexed Processing of Vibrotactile Information in the Mouse Primary Somatosensory Cortex
}

\author{
Yoo Rim Kim ${ }^{1,2,3}$, Chang-Eop Kim ${ }^{1,4}$, Heera Yoon ${ }^{5}$, Sun Kwang Kim ${ }^{5,6 *}$ and Sang Jeong Kim ${ }^{1,2,3 *}$ \\ ${ }^{1}$ Department of Physiology, Seoul National University College of Medicine, Seoul 08826, ${ }^{2}$ Department of Biomedical Sciences, \\ Seoul National University College of Medicine, Seoul 08826, ${ }^{3}$ Neuroscience Research Institute, Seoul National University \\ College of Medicine, Seoul 08826, ${ }^{4}$ Department of Physiology, College of Korean Medicine, Gachon University, Seongnam \\ 13120, ${ }^{5}$ Department of Science in Korean Medicine, Graduate School, Kyung Hee University, Seoul 02447, ${ }^{6}$ Department of \\ Physiology, College of Korean Medicine, Kyung Hee University, Seoul 02447, Korea
}

The primary somatosensory (S1) cortex plays a key role in distinguishing different sensory stimuli. Vibrotactile touch information is conveyed from the periphery to the S1 cortex through three major classes of mechanoreceptors: slowly adapting type 1 (SA1), rapidly adapting (RA), and Pacinian (PC) afferents. It has been a long-standing question whether specific populations in the S1 cortex preserve the peripheral segregation by the afferent submodalities. Here, we investigated whether S1 neurons exhibit specific responses to two distinct vibrotactile stimuli, which excite different types of mechanoreceptors (e.g., SA1 and PC afferents). Using in vivo two-photon microscopy and genetically encoded calcium indicator, GCaMP6s, we recorded calcium activities of S1 L2/3 neurons. At the same time, static $(<1 \mathrm{~Hz})$ and dynamic $(150 \mathrm{~Hz})$ vibrotactile stimuli, which are known to excite SA1 and PC, respectively, were pseudorandomly applied to the right hind paw in lightly anesthetized mice. We found that most active S1 neurons responded to both static and dynamic stimuli, but more than half of them showed preferred responses to either type of stimulus. Only a small fraction of the active neurons exhibited specific responses to either static or dynamic stimuli. However, the S1 population activity patterns by the two stimuli were markedly distinguished. These results indicate that the vibrotactile inputs driven by excitation of distinct submodalities are converged on the single cells of the S1 cortex, but are well discriminated by population activity patterns composed of neurons that have a weighted preference for each type of stimulus.

Key words: Vibrotactile, Mechanoreceptors, Primary somatosensory cortex, Two-photon imaging

\section{INTRODUCTION}

Touch sensation is mainly mediated by three types of mechanoreceptors, slowly adapting type 1 (SA1), rapidly adapting (RA), and Pacinian (PC) afferents $[1,2]$, which are characterized by different response properties. These three types of mechanoreceptors have

Submitted August 31, 2020, Revised October 24,2020,

Accepted October 26, 2020

* To whom correspondence should be addressed.

Sun Kwang Kim, TEL: 82-2-961-0323, FAX: 82-2-961-0333

e-mail:skkim77@khu.ac.kr

Sang Jeong Kim, TEL: 82-2-740-8229, FAX: 82-2-763-9667

e-mail: sangjkim@snu.ac.kr different ranges of sensitivity to various vibrotactile frequencies, and are primarily excited at low $(<5 \mathrm{~Hz})$, intermediate $(5 \sim 50 \mathrm{~Hz})$ and high frequencies $(50 \sim 300 \mathrm{~Hz})$, respectively [3]. It is believed that these afferents respond to different types of skin deformation and serve different functional roles such as shape, texture, and vibration of the touched object [4].

Researchers have been wondering whether there are specific streams for distinct features of sensory stimuli from the periphery to the cortex. Utilizing two-photon imaging technology, it has been explored in the visual, auditory, taste and somatosensory cortex, which mainly focus on how similar but distinct sensory stimuli given at the periphery are separately represented in the sensory cortex [5-8]. In the study of touch sensation, it has been studied
Copyright (c) Experimental Neurobiology 2020. www.enjournal.org
This is an Open Access article distributed under the terms of the Creative Commons Attribution Non-Commercial License (http://creativecommons.org/licenses/by-nc/4.0) which permits unrestricted non-commercial use, distribution, and reproduction in any medium, provided the original work is properly cited. 
whether the information derived from distinct submodalities is segregated or converged at the upper levels of the somatosensory pathway, including the S1 cortex [9-14]. Recently, the principle of convergence has been proposed in a few studies [15-18]. Our previous study has also revealed that the texture feature of touch is selectively encoded in the S1 neurons, whereas S1 neurons are less selective to dynamics feature (static $[<1 \mathrm{~Hz}]$ versus dynamic [1 Hz]) of touch [19]. However, the frequency difference between those stimuli given for dynamics feature in the study was not large enough to activate different types of mechanoreceptors. Therefore, this subject needs to be reevaluated through experiments in which distinct vibrotactile stimuli excite the different types of mechanoreceptors.

In this study, static $(<1 \mathrm{~Hz})$ and dynamic $(150 \mathrm{~Hz})$ vibrotactile stimuli, known as stimuli frequencies to excite SAl and PC, respectively, were used to investigate how distinct vibrotactile stimuli are encoded in the S1 cortex at the single-cell and population levels. Using in vivo two-photon calcium imaging, we found that the majority of the S1 L2/3 neurons were discriminative neurons with weighted responses between the static and dynamic stimuli. Only about 20\% of them were specific neurons that have selective responses to either static or dynamic stimuli. However, the population activity patterns in the S1 cortex were clearly distinguished between the two stimuli. Taken together, these results suggest that the vibrotactile inputs from different types of mechanoreceptors are generally converge on the S1 cortex at the single-cell level, but differentially encoded at the population level through the computation of responses from the preferred neurons as well as the specific neurons.

\section{MATERIALS AND METHODS}

\section{S1 craniotomy and viral injection}

All experimental procedures were approved by the Seoul National University Institutional Animal Care and Use Committee and performed in accordance with the guidelines of the National Institutes of Health. All surgical and viral injection procedures were described in detail in a previous study [19]. Briefly, we used C57BL/6 male mice for in vivo two-photon calcium imaging. A small craniotomy was conducted over the left S1 cortex (size, $2 \times 2$ $\mathrm{mm}$; center relative to Bregma: lateral, 1.5 ; posterior $0.5 \mathrm{~mm}$ ) corresponding to the right hind-paw of the anesthetized mice (isoflurane, 1 1.5\%). The dura was left intact and the animal skull was carefully removed using a \#11 surgical blade. And then we injected adeno-associated virus expressing GCaMP6s (AV-1-PV2824) into the exposed cortex at $2 \sim 3$ sites using a glass pipette $(20 \sim 40 \mu \mathrm{m}$ tip diameter). Finally, the exposed cortex was sealed with a cover glass
(Matsunami, Japan) using Vetbond (3M). Body temperature of the mouse was maintained at $38^{\circ} \mathrm{C}$ using a heating pad (IL-H- 80 , Live Cell Instrument) during all experimental procedures. In vivo calcium imaging was performed two weeks after the surgery.

\section{In vivo two-photon calcium imaging of layer $2 / 3$ neurons in the S1 cortex}

All imaging were conducted in lightly anesthetized mice expressing GCaMP6s. Two-photon calcium imaging was performed with a two-photon microscope (Zeiss LSM 7 MP, Carl Zeiss, Jena, Germany) equipped with a water immersion objective (Apochromat 20×, NA=1.0, Carl Zeiss). Two-photon excitation for GCaMP6s imaging $(900 \mathrm{~nm})$ was supplied by a mode locked Ti: sapphire laser system (Chameleon, Coherent). Imaging data were gained using ZEN software (Zeiss Efficient Navigation, Carl Zeiss). For calcium imaging of the $\mathrm{L} 2 / 3$ neurons, recording was performed at a depth of approximately $200 \mu \mathrm{m}$ from the surface.

\section{Peripheral vibrotactile stimulation}

All stimuli were applied to the right hind-paw of the lightly anesthetized mice while transient calcium activities of S1 neurons were recorded. Static $(<1 \mathrm{~Hz})$ and dynamic $(150 \mathrm{~Hz})$ stimuli were pseudo randomly delivered to the right hind-paw using a custommade vibrotactile stimulator. Static or dynamic stimuli were applied in 6 trials for 5 seconds each. Each stimulus was given in a 20-second interval to minimize sensitization.

\section{Data analysis}

All analysis protocols are consistent with those in our previous study [19]. Regions of interest (ROIs) were manually marked in circles by detecting fluorescence of individual cell bodies in the recorded time-lapse movie. Only soma was included in our analysis. Customized scripts in MATLAB were used to analyze the obtained calcium signals. Calcium signal amplitudes were calculated as $\Delta F /$ $F_{0}\left(\Delta F=F-F_{0}\right)$ for each neuron. $F_{0}$ means the baseline fluorescence signal computed by averaging the lowest $30 \%$ of all fluorescence signals from individual traces. We analyzed responsive neurons only, which were defined as neurons with $\Delta F / F_{0}>30 \%$ to a stimulus. To determine the degree of preference to each stimulus, we calculated preference index (PI) that ranges from 0 to 1 . PI of cell $i$ for stimulus $j\left(P I_{i j}\right)$ was defined as

$$
P I_{i j}=\frac{\overline{P_{l j}}}{\operatorname{Max}_{i}}
$$

where $\overline{P_{l y}}$. is the mean of the peak values of cell $i$ for stimulus $j$ across repeated trials $\left(P_{i j k}\right)$ and $P_{i j k}$ was determined as the highest value of amplitude during each trial $k$ for stimulus $j$ in cell $i$. Max ${ }_{i}$ 
A

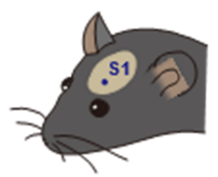

before stimuli
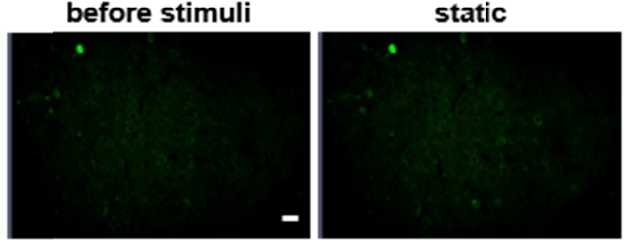

B

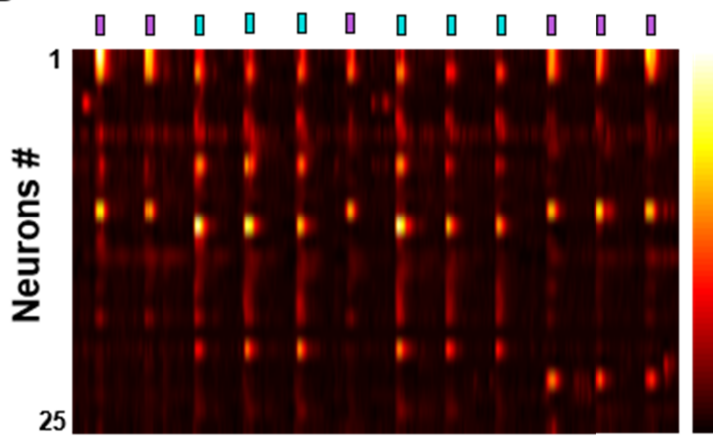

Time (s)

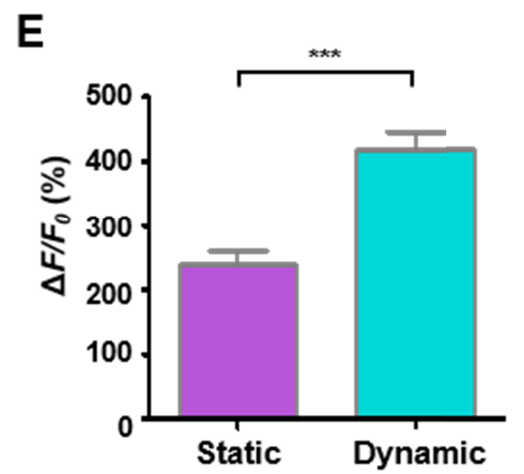

C

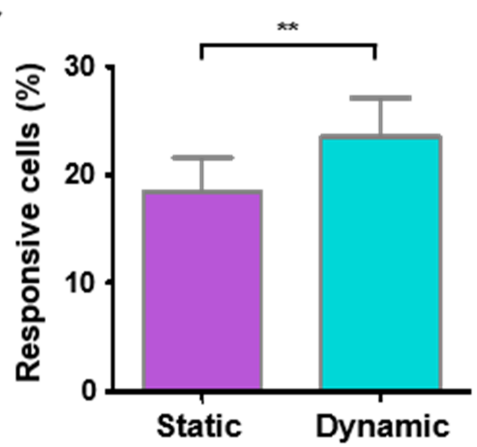

D

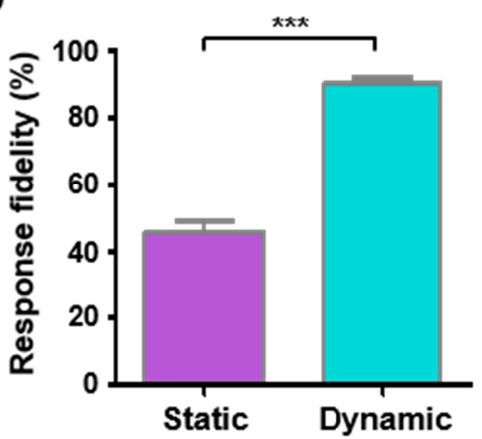

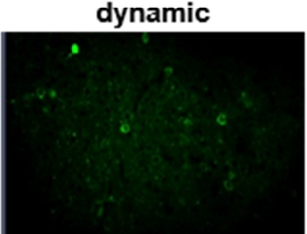

Fig. 1. The neural response properties of S1 L2/3 neurons evoked by static and dynamic stimuli applied to the right hind paw in lightly anesthetized mice. (A) (Top) A craniotomy was conducted over the primary somatosensory cortex (S1) corresponding to the right hind paw in the left hemisphere. In the lightly anesthetized head-fixed mice, static and dynamic stimuli were pseudorandomly applied with a custom-made vibrotactile stimulator, while S1 $\mathrm{L} 2 / 3$ neurons were recorded. (Bottom) Representative images of $\mathrm{Ca}^{2+}$ fluorescence of the S1 L2/3 neurons before and during static or dynamic stimuli. Scale bar, $20 \mu \mathrm{m}$. (B) Representative color-coded raster plots of responsive neurons in response to static or dynamic stimuli in the S1 L2/3. Each colored square box above the raster plots indicates when each static (purple) or dynamic (cyan) stimulus was given. Each stimulus was applied for $5 \mathrm{~s}$. (C) The

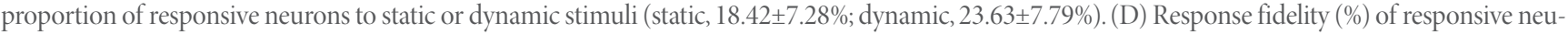
rons to static or dynamic stimuli (static, $45.63 \pm 3.27 \%$; dynamic, $90.21 \pm 2.04 \%$ ). (E) $\Delta F / F_{0}(\%)$ of responsive neurons to static or dynamic stimuli (static, $237.78 \pm 21.24 \%$; dynamic, $418.49 \pm 27.40 \%)$ in S1 L2/3 ( $\mathrm{n}=126$ cells from 5 mice; Two-tailed paired $t$-test, $\left.{ }^{* *} \mathrm{p}<0.01,{ }^{* * *} \mathrm{p}<0.001\right)$. All data are represented as mean \pm s.e.m.).

is the highest value that cell $i$ showed during the experiments. We defined cell $i$ to be "discriminative" or "tuned" to stimulus $j$ when $P I_{i j}$ is larger than $0.8 * \overline{P I_{l}}$, where $\overline{P I_{l}}$ is the mean of $P I_{i j}$ for all the given stimulus. The discriminative neurons were further divided into preferred neurons and specific neurons depending on whether they respond to both types of the stimuli or either stimulus. Principal component analysis (PCA) is a dimensionality reduction method and used in order to represent population activity patterns of S1 L2/3 neurons. N-dimensional activity patterns ( $n$, number of cells) across all recorded time were projected onto their three principal component axes.

\section{Statistics}

All data were analyzed and plotted using custom-written MATLAB scripts (MathWorks) or Prism software (Gragh Pad Software,
USA). To determine the significance in statistical comparisons, Two-tailed paired $t$-test (Fig. 1C E), one-way ANOVA with Tukey's post-hoc test (Fig. 2C and 2D were used. All data are represented as mean \pm s.e.m. The differences were considered significant if a $\mathrm{p}$ value is below 0.05 . NS indicates $\mathrm{p}>0.05$, ${ }^{*}$ indicates $\mathrm{p}<0.05$, ${ }^{\star *}$ indicates $\mathrm{p}<0.01,{ }^{* * *}$ indicates $\mathrm{p}<0.001$.

\section{RESULTS AND DISCUSSION}

We used in vivo two-photon calcium imaging with mice expressing GCaMP6s and primarily examined how distinct vibrotactile stimuli were represented in the $S 1$ cortex. To this end, calcium activities of S1 L2/3 neurons were recorded while static and dynamic stimuli were pseudorandomly applied to the right hind paw in lightly anesthetized mice (Fig. 1A). We found that the S1 L2/3 


\section{A}
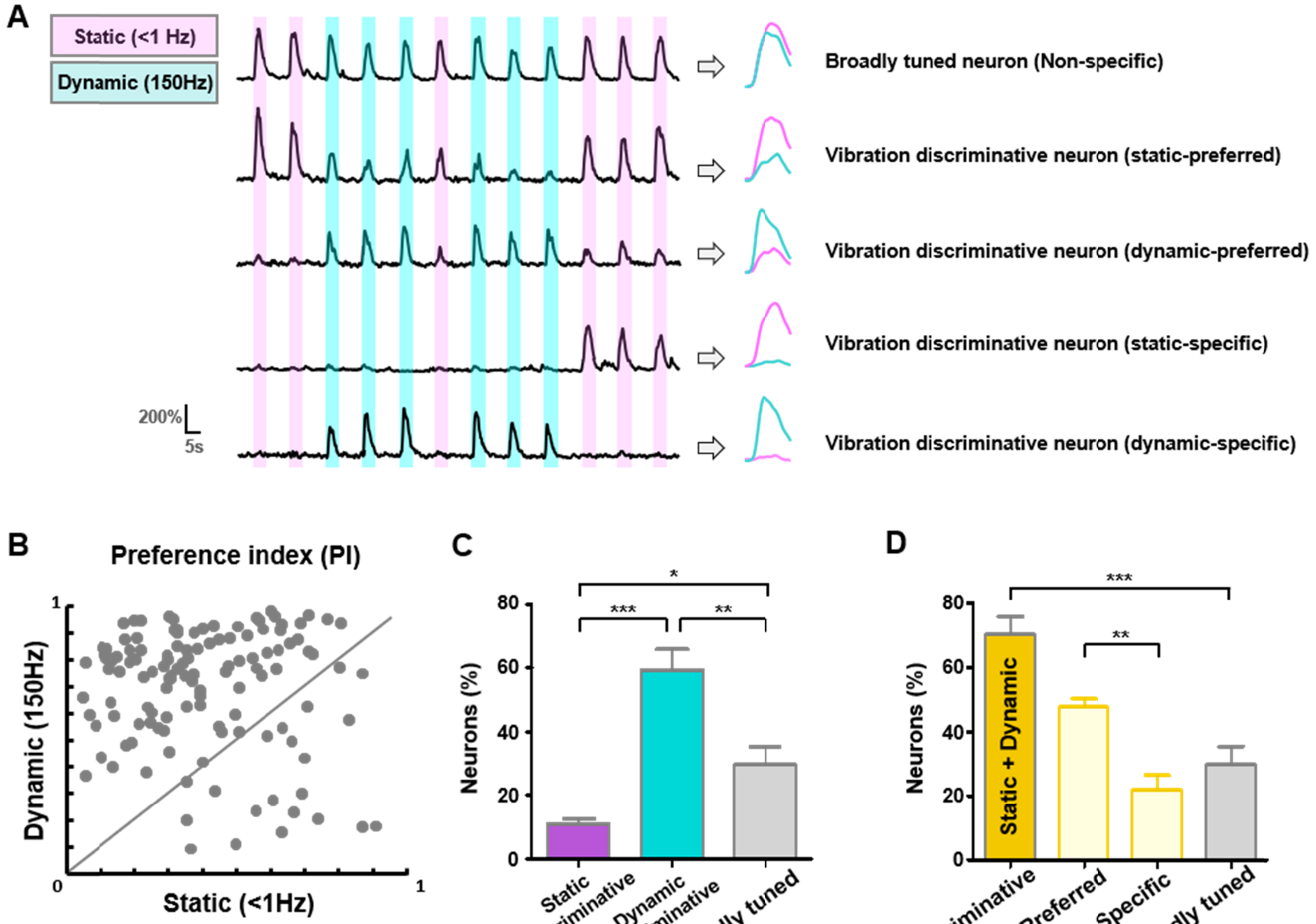

C

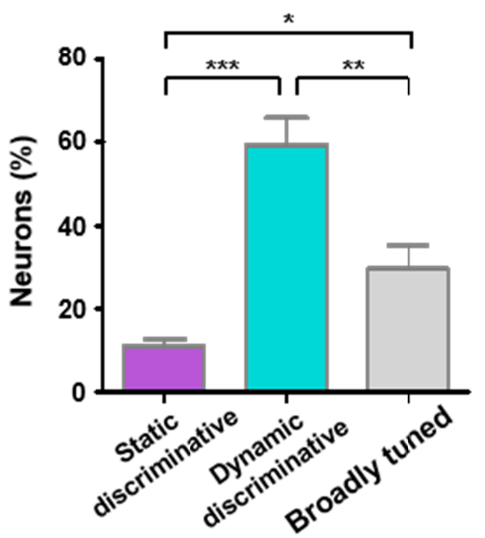

D

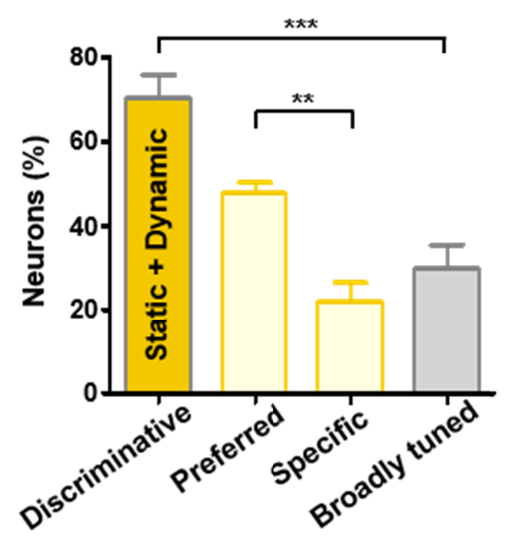

Fig. 2. Discriminative but converged responses of S1 individual neurons to different vibrotactile stimuli. (A) Representative patterns of $\mathrm{Ca}^{2+}$ activities to static and dynamic stimuli from five neurons. Each stimulus was applied in six trials for $5 \mathrm{~s}$ each and marked by vertical lines (purple, static; cyan, dynamic). Purple or cyan traces on the right side of each trace show averaged $\mathrm{Ca}^{2+}$ activity for six trials. The uppermost neuron of the five neurons is a broadly tuned neuron, and the four others below are vibration discriminative neurons. (B) Scatter plots of preference index (PI) of individual neurons for static versus dynamic stimuli ( $\mathrm{n}=126$ cells from 5 mice). (C) The proportions of static discriminative, dynamic discriminative and broadly tuned neurons (static-discriminative, $10.87 \pm 3.85 \%$; dynamic-discriminative, $59.33 \pm 14.52 \%$; broadly-tuned, $29.78 \pm 12.45 \%$ ). (D) The discriminative neurons on the $(\mathrm{C})$ are divided into preferred $(47.66 \pm 5.90 \%)$ and specific neurons $(21.91 \pm 9.91 \%)$. Data are represented as mean \pm s.e.m. Statistics was performed with one-way ANOVA with Tukey's post hoc test, ${ }^{*} \mathrm{p}<0.05,{ }^{* *} \mathrm{p}<0.01,{ }^{* *} \mathrm{p}<0.001$.

neurons responded well to both types of stimuli, but overall responded better to dynamic stimuli (Fig. 1A, B). The proportion of responsive neurons was significantly greater for dynamic stimuli than for static stimuli. Additionally, fidelity and $\Delta F / F_{0}$ were also significantly greater for dynamic stimuli (Fig. 1C E). Although the S1 neurons tended to respond better to dynamic stimuli, neurons with various response patterns were intermingled.

Next, we classified cell types according to the response patterns of the active neurons to static and dynamic stimuli (Fig. 2A). First, there were neurons that showed a similar level of response to both stimuli. However, a large fraction of responsive neurons exhibited better responses to one type of the two stimuli. Next, we calculated the preference index (PI) based on their response fidelity and amplitude to each type of stimulus to quantify the response preference of the individual responsive neurons for static and dynamic stimuli (Fig. 2B). Based on the PI, individual neurons were classified into broadly tuned neurons and vibration discriminative neurons (static or dynamic discriminative neurons) (Fig. 2C). The broadly tuned neurons exhibiting similar levels of response to each type of stimulus occupied approximately $30 \%$. The proportions of static and dynamic discriminative neurons were approximately $10 \%$ and $60 \%$, respectively, and the fraction of dynamic discriminative neurons was significantly higher. These vibration discriminative neurons responded to both static and dynamic 
stimuli but had weighted responses. Next, we further examined the extent to which different vibrotactile information converges in the S1 cortex. To confirm this, all the vibration discriminative neurons were divided into preferred neurons, which responded to both types of stimuli but significantly better to either static or dynamic stimuli, and specific neurons that exhibited selective responses to either type of stimulus (Fig. 2D). The specific neurons accounted for approximately $20 \%$, whereas the proportion of preferred neurons was more than twice (47\%) that of specific neurons. These results suggest that the information of different vibrotactile stimuli is considerably converged in the S1 neurons while maintaining a partial segregation.

Thus far, the results indicate that a large fraction of the neurons receive inputs from both types of mechanoreceptors at the singlecell level. We next examined whether distinct vibrotactile stimuli are distinguished at the $\mathrm{S} 1$ population level. Principal component analysis (PCA) is a dimensionality reduction method and was used to represent population activity patterns of S1 neurons for each type of stimulus (Fig. 3). We found that S1 population activities exhibited clearly distinguished trajectories in projected three dimensions to each kind of stimulus (Fig. 3A). We further identified the population activity patterns of neurons that only responded to static or dynamic stimuli. The population activity patterns of dynamic-preferred neurons (static-preferred neurons) were less separated between different states, particularly for nonpreferred stimuli (Fig. 3C, D). As expected, the population activity patterns of broadly tuned neurons were largely intermingled and indistinguishable from each other given different types of stimuli (Fig. 3B). Taken together, these results indicate that even though distinct frequency information is multiplexed at the single-cell level by the broadly tuned and preferred neurons, it can be clearly discriminated with the S1 population activities produced by preferred neurons having weighted responses for either type of the stimuli.

In this study, we have revealed how static and dynamic stimuli, which excite different types of afferents (static, SA1; dynamic, $\mathrm{PC}$ ), are represented both at the single-cell and population levels in the $\mathrm{S} 1$ cortex. About 30\% of responsive neurons in the S1 L2/3 responded indiscriminately to the static and dynamic stimuli, whereas approximately $70 \%$ exhibited discriminative responses
A

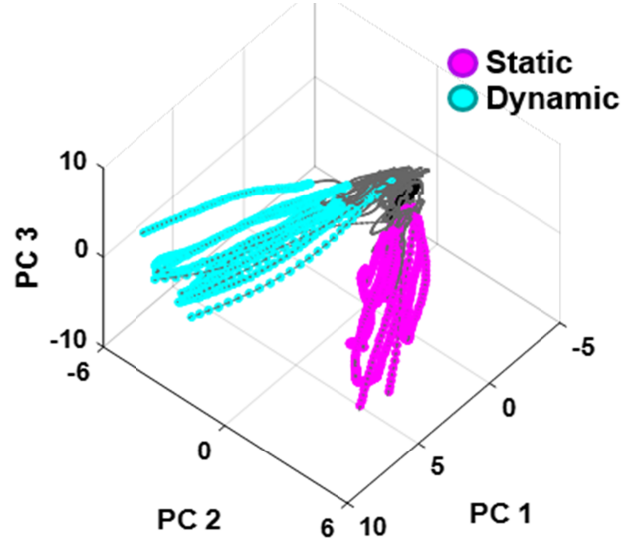

C

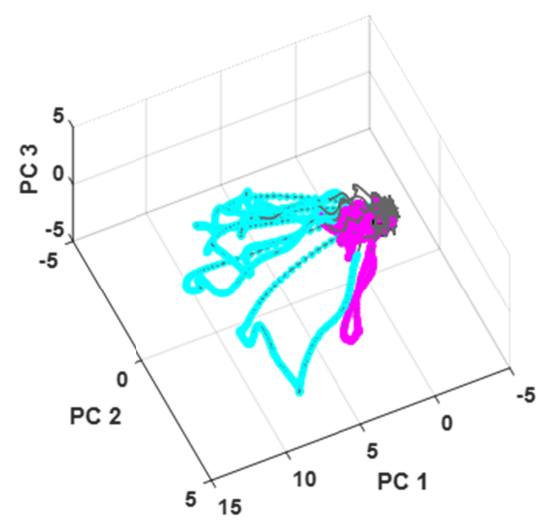

B

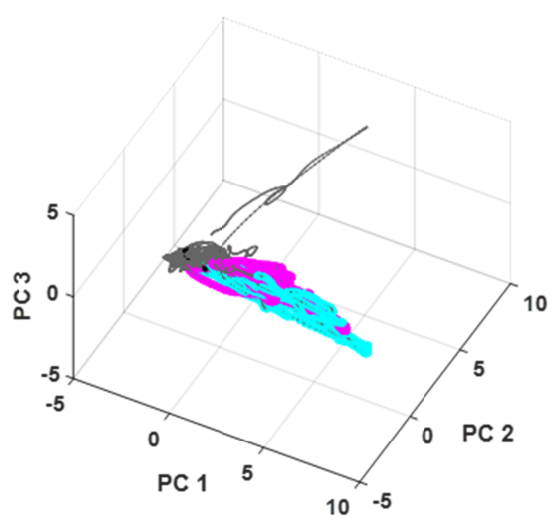

D

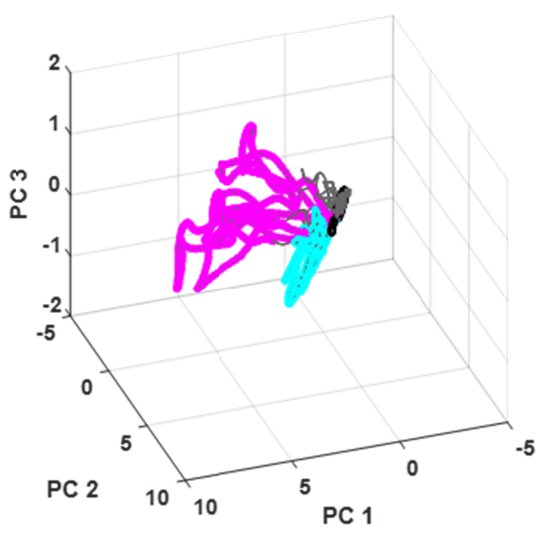

Fig. 3. Population activity patterns of $\mathrm{S} 1$ neurons in response to the static and dynamic stimuli. (A) Using a dimensionality reduction method, population activity patterns of S1 neurons to the static and dynamic stimuli from an example mouse were projected onto their three principal components. Each color (purple, static; cyan, dynamic) corresponds to each type of stimuli. Note that each stimulus evoked distinct activity patterns in the state space. (B) The population activity patterns of broadly tuned neurons were largely intermingled in projected three dimensions given different types of stimuli. (C, D) The population activity patterns of dynamic-preferred neurons (C) or static-preferred neurons (D) were less separated between different states, particularly for nonpreferred stimuli. 


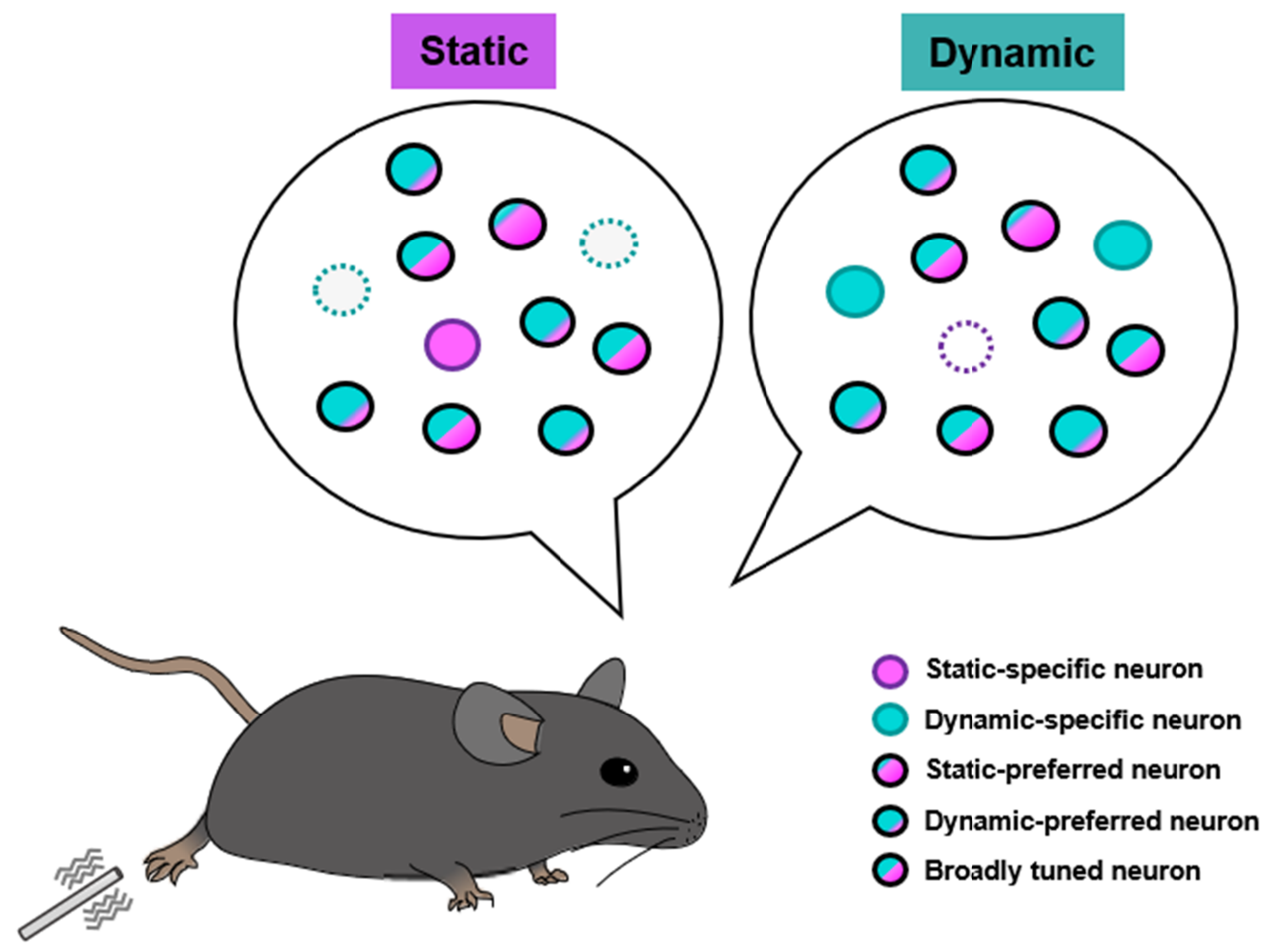

Fig. 4. Multiplexed processing of vibrotactile frequency in the S1 cortex. When the stimuli of different vibrotactile frequencies that excite different types of mechanoreceptors are applied, a few S1 neurons show selective responses to different frequencies. However, a large fraction of the S1 neurons respond to various frequencies, and some of them exhibit equally tuned responses to different frequencies. The rest of the neurons show mixed responses with a weighted preference to a specific type of stimulus. Although the inputs driven by excitations of different afferent classes are multiplexed on the individual neurons in S1, the difference could be discriminated as population activity patterns composed of the preferred neurons and a small portion of specific neurons. Neurons marked with dotted lines represent specific neurons that do not fire when a static (or dynamic) stimulus is given, but only fire when a dynamic (or static) stimulus is given.

between the stimuli. Of the 70\%, however, only $20 \%$ showed selective responses to either type of stimulus. This means that a large fraction of the S1 L2/3 neurons exhibited converged but preferred responses with a weighted preference to either type of the stimuli when static or dynamic stimuli were given (Fig. 4). Nevertheless, distinct vibrotactile stimuli were perfectly distinguished by the $\mathrm{S} 1$ population activity patterns. It implies that the vibrotactile inputs driven by excitation of different submodalities are substantially converged, but are well encoded in the S1 cortex.

It has been a long-time question how vibrotactile information derived by each type of afferent classes with different response properties is represented at each level of the somatosensory pathways. Some studies have argued that submodality-specific streams exist in the somatosensory pathways $[10,12,20]$. In particular, Vernon Mountcastle demonstrated submodality segregation at the $S 1$ cortex by showing S1 neurons being classified as rapidly or slowly adapting. Recent studies, however, have yielded results contrary to the research [18, 21-23]. Pei et al. demonstrated that S1 neurons received mixed input from different afferents classes, and Saal et al. also insisted that stimulus information from RA and PC was intermixed onto S1 individual cells. In line with these results, our study showed that the vibrotactile information driven by excitations of different submodality classes corresponding to low and high frequency, respectively, is multiplexed onto the $S 1$ individual neurons. These accumulated results imply that the inputs of each type of stimulus from the periphery are not preserved in the cortex, but rather converge into individual cells and are distinguished as the different perception by the ensemble activity patterns of S1 neurons. Saal et al. [18] revealed that intermixed information in the cortical neurons influences distinct aspects of responses of the $\mathrm{S} 1$ neurons. They reported that RA input affected the spike rate of $\mathrm{S} 1$ responses, but PC input affected the spike timing and excitationinhibition balance. Zuo et al. [24] also demonstrated that the information in the spike timing of the neurons conveys more texture information, but the spiking rate and timing are complementarily multiplexed in the somatosensory cortex for perceptual decisions. This phenomenon could be an optimal strategy for the limited capacity of cortical neurons to efficiently process inputs by multiple 
afferents, which are simultaneously activated by natural stimuli encountered in the environment.

Our previous study reported how texture, dynamics and noxiousness features of touch and pain are encoded at the single-cell and population levels in the S1 cortex [19]. We showed that S1 neurons exhibited nonselective responses to dynamics feature. However, it was difficult to assume that the given stimuli were mediated by different mechanoreceptors at the periphery. We redeemed that in this study and applied two types of vibrotactile stimuli that have a large difference in the frequency of vibration and are known to excite SA1 and PC, respectively. As a result, we reaffirmed that tactile information by different submodality classes is collectively conveyed to the $\mathrm{S} 1$ neurons in a convergent manner, even though a few specific neurons exist.

If this is so, how are the segregated peripheral inputs converged at the upper levels, including the S1 cortex? One of the possible mechanisms was proposed by Sakurai et al. [16], showing that axonal projections of distinct types of touch sensory neurons converge on the same second-order projection neurons in the brainstem. They hypothesized that mixed and preferred signals already occurred in the projection neurons, which coincidentally detected the transient synchronous spikes between various sensory neurons with different response properties. It is possible that the S1, downstream of the submodality-specific afferent neurons, processes more abstract aspects of the somatosensory information given the general principles of information processing of the brain or braininspired artificial neural network such as a convolutional neural network [25]. It is an interesting subject to be discussed in the next study as to how and why the information exhibiting distinct functional response properties appears in a convergent manner, rather than being conveyed and processed by specific neurons in the $\mathrm{S} 1$ cortex.

\section{ACKNOWLEDGEMENTS}

This study was supported by National Research Foundation of Korea grants funded by the Korea government to YRK (NRF2020R1I1A1A01065791), to SJK (NRF-2018R1A5A2025964 and NRF-2017M3C7A1029611), and to SKK (NRF2017M3C7A1025604).

\section{REFERENCES}

1. Johnson KO (2001) The roles and functions of cutaneous mechanoreceptors. Curr Opin Neurobiol 11:455-461.

2. Roudaut Y, Lonigro A, Coste B, Hao J, Delmas P, Crest M (2012) Touch sense: functional organization and molecular determinants of mechanosensitive receptors. Channels (Austin) 6:234-245.

3. Johansson RS, Landström U, Lundström R (1982) Responses of mechanoreceptive afferent units in the glabrous skin of the human hand to sinusoidal skin displacements. Brain Res 244:17-25.

4. Delmas P, Hao J, Rodat-Despoix L (2011) Molecular mechanisms of mechanotransduction in mammalian sensory neurons. Nat Rev Neurosci 12:139-153.

5. Stettler DD, Axel R (2009) Representations of odor in the piriform cortex. Neuron 63:854-864.

6. Chen X, Gabitto M, Peng Y, Ryba NJ, Zuker CS (2011) A gustotopic map of taste qualities in the mammalian brain. Science 333:1262-1266.

7. Garion L, Dubin U, Rubin Y, Khateb M, Schiller Y, Azouz R, Schiller J (2014) Texture coarseness responsive neurons and their mapping in layer 2-3 of the rat barrel cortex in vivo. Elife 3:e03405.

8. Rothschild G, Nelken I, Mizrahi A (2010) Functional organization and population dynamics in the mouse primary auditory cortex. Nat Neurosci 13:353-360.

9. Johnson KO, Hsiao SS (1992) Neural mechanisms of tactual form and texture perception. Annu Rev Neurosci 15:227-250.

10. Sur M, Wall JT, Kaas JH (1981) Modular segregation of functional cell classes within the postcentral somatosensory cortex of monkeys. Science 212:1059-1061.

11. Mountcastle VB (1957) Modality and topographic properties of single neurons of cat's somatic sensory cortex. J Neurophysiol 20:408-434.

12. Ochoa J, Torebjörk E (1983) Sensations evoked by intraneural microstimulation of single mechanoreceptor units innervating the human hand. J Physiol 342:633-654.

13. Dykes RW, Sur M, Merzenich MM, Kaas JH, Nelson RJ (1981) Regional segregation of neurons responding to quickly adapting, slowly adapting, deep and Pacinian receptors within thalamic ventroposterior lateral and ventroposterior inferior nuclei in the squirrel monkey (Saimiri sciureus). Neuroscience 6:1687-1692.

14. Dykes RW, Rasmusson DD, Sretavan D, Rehman NB (1982) Submodality segregation and receptive-field sequences in cuneate, gracile, and external cuneate nuclei of the cat. J Neurophysiol 47:389-416.

15. Saal HP, Bensmaia SJ (2014) Touch is a team effort: interplay of submodalities in cutaneous sensibility. Trends Neurosci 37:689-697.

16. Sakurai K, Akiyama M, Cai B, Scott A, Han BX, Takatoh J, Sigrist M, Arber S, Wang F (2013) The organization of submo- 
dality-specific touch afferent inputs in the vibrissa column. Cell Rep 5:87-98.

17. Harvey MA, Saal HP, Dammann JF 3rd, Bensmaia SJ (2013) Multiplexing stimulus information through rate and temporal codes in primate somatosensory cortex. PLoS Biol 11:e1001558.

18. Saal HP, Harvey MA, Bensmaia SJ (2015) Rate and timing of cortical responses driven by separate sensory channels. Elife 4:e10450.

19. Kim YR, Kim CE, Yoon H, Kim SK, Kim SJ (2019) S1 employs feature-dependent differential selectivity of single cells and distributed patterns of populations to encode mechanosensations. Front Cell Neurosci 13:132.

20. Sur M, Wall JT, Kaas JH (1984) Modular distribution of neurons with slowly adapting and rapidly adapting responses in area $3 \mathrm{~b}$ of somatosensory cortex in monkeys. J Neurophysiol 51:724-744.
21. Pei YC, Denchev PV, Hsiao SS, Craig JC, Bensmaia SJ (2009) Convergence of submodality-specific input onto neurons in primary somatosensory cortex. J Neurophysiol 102:18431853.

22. Pei YC, Hsiao SS, Craig JC, Bensmaia SJ (2010) Shape invariant coding of motion direction in somatosensory cortex. PLoS Biol 8:e1000305.

23. Dépeault A, Meftah el-M, Chapman CE (2013) Neuronal correlates of tactile speed in primary somatosensory cortex. J Neurophysiol 110:1554-1566.

24. Zuo Y, Safaai H, Notaro G, Mazzoni A, Panzeri S, Diamond ME (2015) Complementary contributions of spike timing and spike rate to perceptual decisions in rat $\mathrm{S} 1$ and $\mathrm{S} 2$ cortex. Curr Biol 25:357-363.

25. Yamins DL, DiCarlo JJ (2016) Using goal-driven deep learning models to understand sensory cortex. Nat Neurosci 19:356-365. 